\title{
Voltage disturbance detection method for HTS tape using electromagnetically coupled coils
}

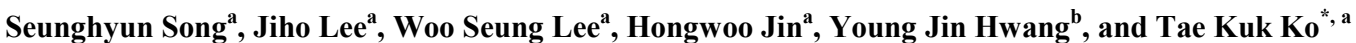 \\ ${ }^{a}$ Yonsei University, Seoul, Korea \\ ${ }^{\mathrm{b}}$ Korea Evaluation Institute of Industrial Technology
}

(Received 5 February 2014; revised or reviewed 19 March 2014; accepted 20 March 2014)

\begin{abstract}
This paper represents the detection method of voltage disturbance for high temperature superconducting (HTS) tape using electromagnetically coupled coils. In order to detect the voltage as the superconductor transits from the superconducting state to the normal conduction state, voltage taps are widely used to get the voltage signal. And voltage taps are connected to data acquisition device via signal wires. However this new suggested method can detect the superconducting transition voltage without signal wires between voltage taps and data acquisition device by using electromagnetically coupled coils. This system consists of two electromagnetically coupled coils, the first coil to detect and transmit the voltage of HTS tape and the second coil to pick up the transmitted voltage from the first coil. By using this new suggested method, we can build the 'separated voltage-detection system'. HTS tape and first coil are located under liquid nitrogen vessel and the second coil is located under room temperature condition. In this paper, experiments are performed to verify the feasibility of the proposed method. As the result of the experiment, the separated voltage-detection system using electromagnetically coupled coils can successfully observe superconducting-normal transition of HTS tapes.
\end{abstract}

Keywords: coil, detection, electromagnetic induction, superconducting-normal transition, voltage tap, wireless

\section{INTRODUCTION}

To detect the superconducting-normal transition voltage of high temperature superconducting (HTS) tapes, physically connected signal wires for voltage taps are typically used [1]. However in some cases, signal wires for voltage taps are useless or even worse, they cause the significant problem to detect the voltage [2]. Therefore, in this paper, we suggest the new method, 'separated voltage-detection system' for HTS tape using electromagnetically coupled coils. This method consists of two electromagnetically coupled coils. One is called 'first coil' and detects and transmits the voltage of HTS tape, and the other is called 'second coil' and picks up the transmitted voltage from first coil. When superconducting-normal transition occurs, it is possible to transmit and receive the voltage, because the dramatic change of the voltage is appeared and it is not constant component. The first coil and HTS tape are under the liquid nitrogen vessel and the second coil is under room temperature condition. In this study, voltage detection experiments are performed and this new method succeed in the detection of superconducting-normal transition of HTS tape. Authors expect that this new method can be applied to superconducting wind generator. Most of the superconducting wind generator needs the voltage taps for the quench detection of the rotor coil [3]. However, as the superconducting rotor coil rotates in order to generate the magnetic flux, signal wires are twisted. So, the twist of wires for voltage taps could be an issue for superconducting rotating machine [4]. However, by

* Corresponding author: tkko@yonsei.ac.kr separating the detection part from the superconducting rotor coil by means of electromagnetic coupling, the problem can be solved. As the result, this wireless system can be applied to the superconducting wind generator.

\section{EXPERIMENT}

\subsection{Theoretic background}

If two coils which have $N_{l}$ and $N_{2}$ turns respectively are wound with copper, and the current $I_{l}$ flows through the first coil, the electromagnetic induction occurs between two coils. The magnetic flux generated by the first coil penetrates through the second coil. And by the penetrated magnetic flux, the electromotive force is generated at the second coil. As the result, the transition voltage of the HTS tape can be detected by using the electromotive force. Fig. 1 is the schematic drawing for electromagnetic induction system.

\subsection{Experimental set-up}

Table I and II show the specifications of HTS tape and two electromagnetically coupled coils used in this study. The first coil has 240 turns and is connected to the HTS tape in parallel. The first coil and the HTS tape are located under the same liquid nitrogen vessel. HTS voltage is proportional to the current which flows through the HTS tape as the HTS tape transits from the superconducting state to the normal conducting state. Since the HTS tape and the first coil are connected in parallel, HTS tape and the first coil have the same magnitude of the voltage. 


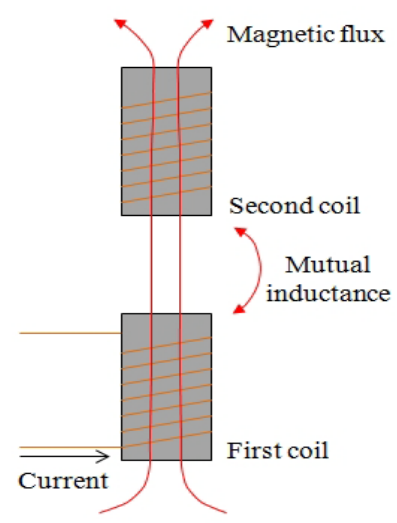

Fig. 1. Schematic drawing for electromagnetic induction system.

TABLE I

SPECIFICATIONS OF THE HTS TAPE.

\begin{tabular}{cc}
\hline Parameters & Values \\
Manufacturer & American Superconductor \\
Product & $344 \mathrm{~s}$ \\
Total length & $30 \mathrm{~cm}$ \\
Voltage tap length & $10 \mathrm{~cm}$ \\
Critical current & $81 \mathrm{~A}$ \\
\hline
\end{tabular}

TABLE II

SPECIFICATIONS OF THE COILS.

\begin{tabular}{ccc}
\hline & $1^{\text {st }}$ coil & $2^{\text {nd }}$ coil \\
Conductor type & Copper & Copper \\
Turns & 240 turns & 470 turns \\
Inductance & $6.53 \mathrm{mH}$ & $28.26 \mathrm{mH}$ \\
\hline
\end{tabular}

The second coil has 470 turns and isolated from the liquid nitrogen vessel. As the first coil and the second coil are connected magnetically, the magnetic flux interlinks between two coils. Therefore, in accordance with Faraday's law the electromotive force occurs in the second coil as the current of HTS tape is increased. Fig. 3 shows the schematic drawing of separated voltage-detection system. Variables of test are separation distance between the first coil and the second coil and the ramping rate of the current. American Superconductor (AMSC)'s 344s is used as the test sample. The distance between voltage taps is $10 \mathrm{~cm}$. As a solder to attach the HTS tape on the copper terminal blocks, $\mathrm{In}_{52} \mathrm{Sn}_{48}$ alloy is used

\subsection{Experimental results and analysis}

In order to determine the critical current $\left(I_{c}\right)$, the criterion of $1 \mu \mathrm{V} / \mathrm{cm}$ is used. Therefore, the criterion of superconducting transition when the superconductor transits from the superconducting state to the normal conducting state is $10 \mu \mathrm{V}$ and $I_{c}$ of the HTS tape is $81 \mathrm{~A}$ as shown below figures. Totally eight tests are performed according to the distance between the first and the second coil and ramping rate of the current. Self-inductances of the first coil and the second coil are $6.53 \mathrm{mH}$ and $28.26 \mathrm{mH}$, respectively. As shown in Fig 5, the first coil's voltage and

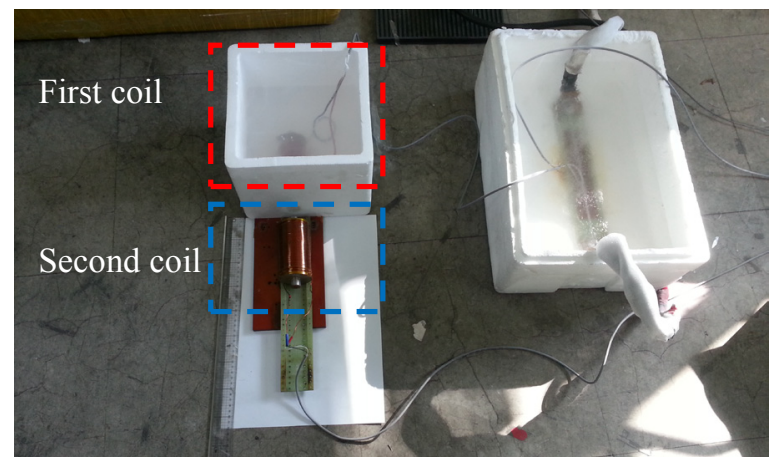

Fig. 2. Experimental set up of the HTS tape.

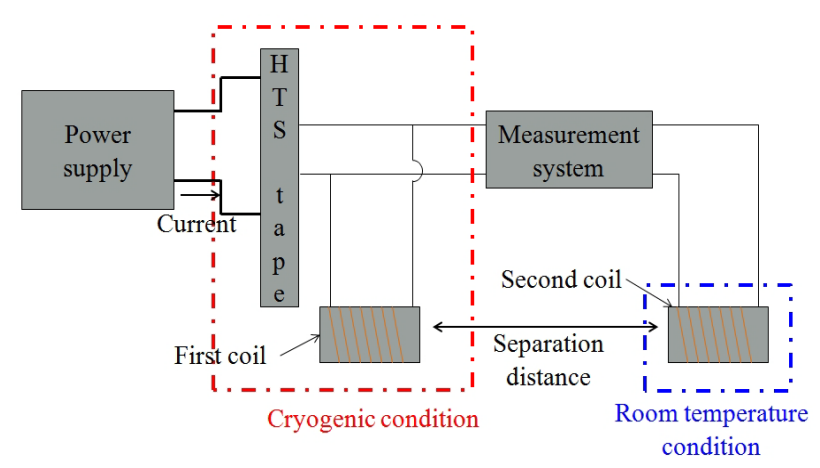

Fig. 3. Schematic drawing of HTS voltage detection system.

the second coil's voltage almost concurrently increase. The shape of the V-I curve is similar to those of the result of Fig. 4 when the ramping rate is $2 \mathrm{~A} / \mathrm{s}$. In order to analyze the test result, the average values of the second coil voltages from 0 to $50 \mathrm{~s}$ with $1 \mathrm{~A} / \mathrm{s}$ ramping rate and from 0 to $25 \mathrm{~s}$ with $2 \mathrm{~A} / \mathrm{s}$ ramping rate are calculated. When the ramping rate of current is $1 \mathrm{~A} / \mathrm{s}$, the average values of the second coil voltages are increased from 0.1998 to $3.0530 \mu \mathrm{V}$ as the separated distances are increased from $2 \mathrm{~cm}$ to $8 \mathrm{~cm}$ with $2 \mathrm{~cm}$ increment. To analyze the effect of the separated distance between two coils on the magnitude of the voltage of the second coil, voltage increments during superconducting-normal transition are calculated based on the transition criterion, $10 \mu \mathrm{V}$. Voltage increments are calculated by subtracting the second coil's voltage from the first coil's voltage when the first coil's voltage reaches the transition criterion. The test result shows the tendency that voltage increment during superconducting-normal transition is inverse proportion to the separated distance. During the superconducting-normal transition, if the voltage increment is high enough, fast voltage detection of HTS wire can be performed. In other words, the higher voltage increment is, the faster the voltage detection time is. Moreover, as the ramping rate of current is increased, the voltage increment is also increased. For example, when the ramping rate of current is $1 \mathrm{~A} / \mathrm{s}$, the voltage differences are decreased from 0.1998 to $0.0229 \mu \mathrm{V}$ as the separation distances are increased from $2 \mathrm{~cm}$ to $8 \mathrm{~cm}$. Likewise, when the ramping rate of current is $2 \mathrm{~A} / \mathrm{s}$, the voltage differences 


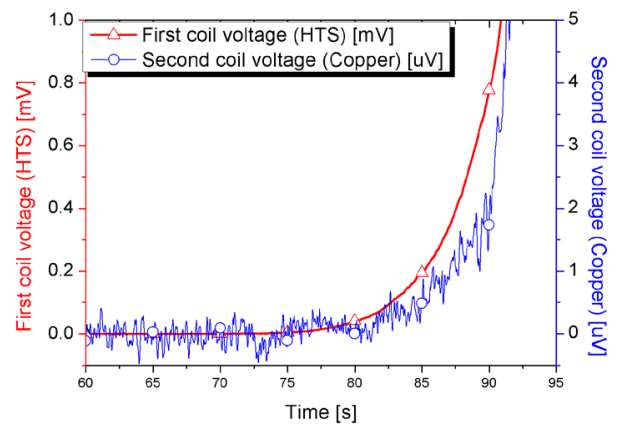

(a)

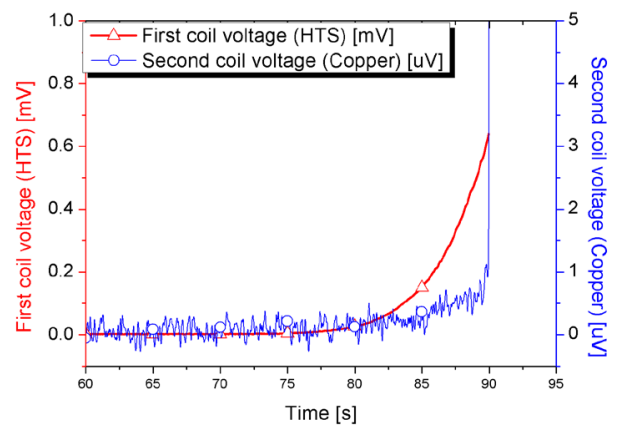

(b)

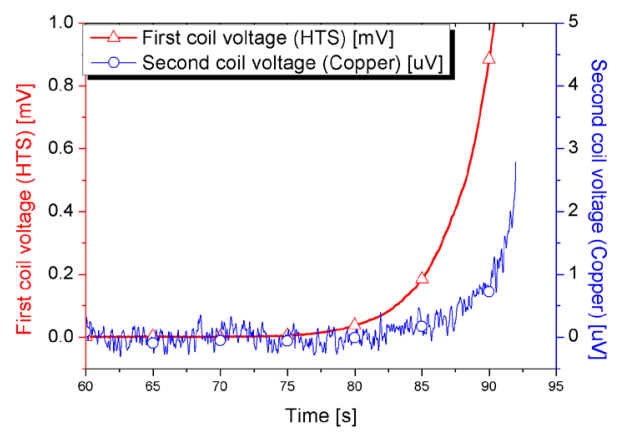

(c)

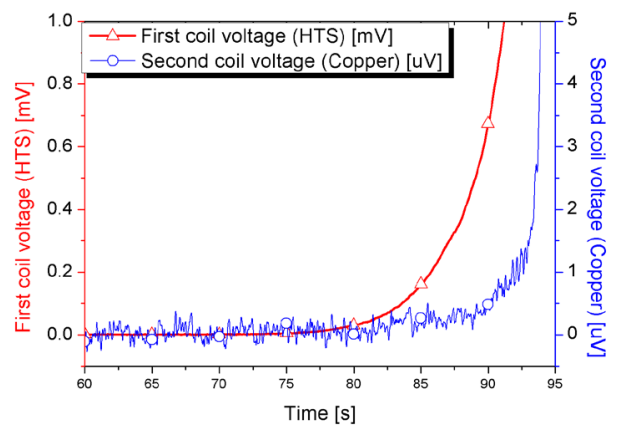

(d)

Fig. 4. V-I curve of the first coil and the second coil voltages with ramping rate of $1 \mathrm{~A} / \mathrm{s}$ :

(a) the separation distance is $2 \mathrm{~cm}$

(b) the separation distance is $4 \mathrm{~cm}$

(c) the separation distance is $6 \mathrm{~cm}$

(d) the separation distance is 8

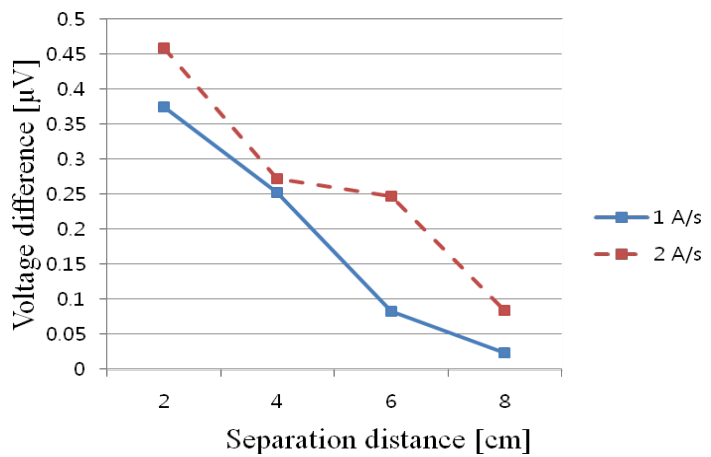

Fig. 5. The results of the voltage difference when the ramping rates of current are $1 \mathrm{~A} / \mathrm{s}$ and $2 \mathrm{~A} / \mathrm{s}$.

are decreased from 2.7364 to $0.0841 \mu \mathrm{V}$ as the separation distances are increased from $2 \mathrm{~cm}$ to $8 \mathrm{~cm}$. As a result, the voltage detection time is getting faster, as the separation distance is shorter and the current ramping rate is higher.

\section{CONCLUSION}

In this paper, the new detection method of voltage disturbance of HTS tape using electromagnetically coupled coils is introduced. Generally, to detect the HTS voltage of superconducting state to normal state transition, voltage taps are widely used to get the voltage signal. However by using the wireless voltage detecting technique, HTS voltage can be detected. In order to confirm the reliability of this suggested technique, several cases of the tests are performed according to the separation distance between the first coil and the second coil and ramping rate of the current which flows through the HTS tape. The test results show that the voltage detection time is getting faster, as the separation distance is shorter and the current ramping rate is higher. Therefore, authors expect that this wireless system can be applied to the superconducting wind generator by adjusting the separation distance between the first coil and the second coil.

\section{ACKNOWLEDGMENT}

This work was supported by the Human Resources Development of the Korea Institute of Energy Technology Evaluation and Planning (KETEP) grant funded by the Korean government Ministry of Trade, Industry \& Energy (20124030200040).

\section{REFERENCES}

[1] A.B. Abrahamsen et al., "Feasibility study of 5MW superconducting wind turbine generator" Physica C471, 1464-1469 (2011)., submitted for publication.

[2] H. Kazushi and N. Mariko "Quench detector of superconducting rotary electric machine," Japan Patent 08075 810, May 22, 1996. 
[3] D.T. Ryan et al., "Quench monitoring and control system and method of operating same," U.S. Patent 7053509 B2, May 30, 2006.

[4] Stephen D. Umans, Boris A. Shoykhet, "Quench in high-temperature superconducting motor field coils: Experimental results," IEEE T. Ind. Appl., vol. 42, no. 4, July/Aug 2006. Submitted for publication. 